\title{
Body Mass Index Influence on the Clinical Outcomes for Nonvalvular Atrial Fibrillation Patients Admitted to a Hospital Treated with Direct Oral Anticoagulants: A Retrospective Cohort Study
}

\section{Xiaoye Li* \\ Chengchun Zuo* \\ Qiuyi Ji \\ Ying Xue \\ Zi Wang \\ Qianzhou Lv $\mathbb{D}$}

Department of Pharmacy, Zhongshan Hospital, Fudan University, Shanghai,

People's Republic of China

*These authors contributed equally to this work
Correspondence: Qianzhou Lv Department of Pharmacy, Zhongshan Hospital Fudan University, Shanghai, People's Republic of China

Tel +86-2I-64041990

Fax +86-2I-34160880

Email13916088938@I63.com
Background: Considering that the current fixed dose of direct oral anticoagulants (DOACs) might have insufficient anticoagulation effect for overweight patients, the aim of this study was to compare the effectiveness and safety of anticoagulation between dabigatran and rivaroxaban in different body mass index (BMI) population.

Methods: We conducted a retrospective cohort study of 2402 DOAC anticoagulated patients with atrial fibrillation who underwent catheter ablation (1290 dabigatran, 53.7\% and 1112 rivaroxaban, 46.3\%) between January 2017 and December 2018. Patients were distributed based on the BMI into nonobese (1362, BMI $<25 \mathrm{~kg} / \mathrm{m}^{2}$ ), preobese (521, BMI 25.0-29.9 kg/ $\mathrm{m}^{2}$ ), class I obese $\left(344\right.$, BMI $\left.30.0-34.9 \mathrm{~kg} / \mathrm{m}^{2}\right)$ and class II+ obese $\left(175, \mathrm{BMI} \geq 35.0 \mathrm{~kg} / \mathrm{m}^{2}\right)$. We collected information regarding clinical features, laboratory data, bleeding complications and systemic embolic events from the electrical medical records system during 12 months. Results: The incidence of systemic embolism and stroke complications was higher in the class II+ obese group ( $P=0.001$ and $P=0.003)$. The incidence of bleeding complications and the levels of anticoagulation parameters under the bleeding threshold were similar among the four groups $(P>0.05)$. Cumulative Kaplan-Meier analysis illustrated that rivaroxaban-treated patients who belonged to higher BMI subgroups were more likely to experience shorter time to thrombosis (TTT) (12-month TTT rates of $0.5 \%$ for nonobese vs $1.7 \%$ for class I obese patients, $\mathrm{HR}=3.716, P=0.005 ; 12$-month TTT rates of $0.5 \%$, for nonobese vs $4.0 \%$ for class II + obese patients, $\mathrm{HR}=6.843, P=0.001$ ). However, no statistical significant difference in terms of the time to bleeding complications and the time to cumulative events among the four groups was observed. By multivariate analysis, a higher BMI value (BMI $\geq 25 \mathrm{~kg} / \mathrm{m}^{2}$ ) $(P=0.019 ; \mathrm{OR}=2.094,95 \% \mathrm{CI}: 1.129-3.883)$ was an independent predictor for thrombosis in patients treated with dabigatran or rivaroxaban. Positive linear relationship was observed between BMI levels and occurrence rate of thrombosis and bleeding in under anticoagulation patients with NVAF $\left(\mathrm{R}^{2}=0.451\right.$ and $\mathrm{R}^{2}=0.383$, respectively).

Conclusion: The fixed dose of $15 \mathrm{mg}$ rivaroxaban might carry a risk of under exposure, which would lead to an increase of thromboembolic complications in patients with high BMI. Therefore, rivaroxaban dose increase was suggested for obese patients. Use of DOACs appears to have considerable safety in obese patients.

Keywords: body mass index, direct oral anticoagulants, thrombosis, bleeding, composite cardiovascular endpoints 


\section{Introduction}

The direct oral anticoagulants (DOACs), including dabigatran and rivaroxaban, have been widely used for nonvalvular atrial fibrillation (NVAF) patients and are listed as the first choice for anticoagulation by the current guidelines. ${ }^{1}$ DOACs, which are generally not necessary for regular monitoring the coagulation function, have a similar therapeutic effect to warfarin in preventing thrombus and could significantly reduce the incidence of severe bleeding and fatal organ bleeding. ${ }^{2}$

Contrary to the individualized warfarin dosage that was mainly based on the international normalized ratio (INR), DOACs are administrated with fixed doses and could be much more convenient for clinical application without routine coagulation parameters testing. Previous studies indicated that DOACs anticoagulation effect would be dependent on the age, weight, renal function, and concomitant medications. ${ }^{3}$ Considerations of current fixed DOAC dose in the overweight patient failed to achieve therapeutic blood concentration that increased the risk of embolism, ${ }^{4}$ and it raised concerns about their effectiveness in overweight patients with NVAF. The population with a higher body mass index (BMI) accompanied with a larger body surface area and lower dabigatran plasma concentration had an insufficient anticoagulation effect, which might contribute to higher risk of ischemic stroke. $^{5-7}$

Recent, previous studies indicated that DOACs provided consistent effectiveness and safety for obese patients as well; however, evidently, conclusions from these studies were limited by varying weight cut-offs and BMI stratifications. ${ }^{8,9}$ This leads to an urgent need to evaluate the impact of BMI on thrombosis and bleeding outcomes in unselected patients treated with DOACs under realworld conditions.

One fact should also be noted: some large clinical trials that involved DOACs (including dabigatran, apixaban, rivaroxaban, edoxaban) lacked BMI information, which cannot reveal the impact of BMI on the results and the difference of mean BMI among patients. This made the literature more heterogeneous. ${ }^{10}$ Since DOACs are expected to be increasingly used, more information about effectiveness and safety, especially among the overweight that might be exposed to suboptimal drug levels is urgently needed. The purpose of this study was to compare the impact of BMI on the effectiveness and safety of dabigatran or rivaroxaban in NVAF patients.

\section{Method}

\section{Study Design}

We retrospectively reviewed the charts of 2402 consecutive patients, calculated BMI for each patient upon admission based on documented height and weight, and collected the data through the electronic medical records system. We compared procedural and mid-term outcomes of patients who underwent catheter ablation. According to the World Health Organization (WHO) definition of obesity (World Health Organization, 2000) (nonobese with BMI $\leq 24.9 \mathrm{~kg} / \mathrm{m}^{2}$, preobese with BMI $25.0-29.9 \mathrm{~kg} / \mathrm{m}^{2}$, class I obese with BMI $30.0-34.9 \mathrm{~kg} / \mathrm{m}^{2}$, class II+ obese with BMI $\left.\geq 35.0 \mathrm{~kg} / \mathrm{m}^{2}\right),{ }^{11}$ the participants were stratified into four groups. We analyzed the impacts of BMI on the effectiveness and safety of treatment with lower doses of DOACs with either dabigatran or rivaroxaban. The retrospective study was approved by the Medical Ethics Committee of Zhongshan Hospital and was carried out in accordance with the principles of the Declaration of Helsinki (approval number B2021-030). The requirement for informed consent was waived by the ethics committee because this retrospective analysis was limited to preexisting data from medical records and collected as a part of the standard treatment by physicians. Identified data was anonymized and privacy issues were kept confidential.

\section{Patients Information}

We conducted this retrospective cohort study to investigate impact of BMI on the effectiveness and safety of DOACs among patients who were diagnosed as NVAF in the Department of Cardiology, Zhongshan Hospital, Fudan University from January 2017 to December 2018. According to the European Society of Cardiology (ESC) criteria, ${ }^{1}$ NVAF was diagnosed. All the patients were newly presented with the electrocardiogram (ECG) showing a typical pattern of AF: absolutely irregular RR intervals, with no discernible and distinct $P$ waves. The profile of patient enrollment in this study include a higher risk for stroke, transient ischemic attack, and systemic embolism with $\mathrm{CHA}_{2} \mathrm{DS}_{2}$-VASc score $\geq 1$ and medication usage of either dabigatran (110 $\mathrm{mg}$ bid, the only available dosage) or rivaroxaban (15 mg qd) as a part of stroke/systemic embolism risk reduction management. Patients who met one or more of the following criteria were to be excluded from the study: is less than 18 years; was allergic or has contraindications to either dabigatran or rivaroxaban; has the history of major or clinical relevant nonmajor bleeding 
and hemorrhagic disease; severe renal dysfunction; discontinuation of a treatment or treatment switching between dabigatran and rivaroxaban during 12-month follow-ups.

\section{Data Collection}

Detailed information, including history of smoking or alcohol consumption, comorbidities, levels of hemoglobin $(\mathrm{Hb})$ and platelet (PLT) count, alanine aminotransferase of each patient was collected through the electronic medical records system. Estimated glomerular filtration rate (eGFR) was calculated based on the MDRD formula and the study collected cardiac biomarkers as well as concomitant drugs in use. Predetermined stroke and bleeding risk was assessed using the $\mathrm{CHA}_{2} \mathrm{DS}_{2}-\mathrm{VAS}_{\mathrm{C}}$ and HASBLED score. $^{2}$

\section{Medication and Radio Frequency Ablation Procedure}

All the patients had been treated with DOACs for at least four consecutive weeks to ensure a steady anticoagulation state and were admitted to our hospital for scheduled catheter ablation. Upon admission, after taking ECG to confirm no sign of cardiac thrombosis, the patients were scheduled for catheter ablation.

The patients stopped the use of DOACs $24 \mathrm{~h}$ before intervention and resumed DOAC therapy three to four hours after sheath removal. Transesophageal echocardiography was applied based on the operator's preference. According to the operation protocol, the patients received intravenous unfractionated heparin to maintain an activated clotting time from 300 to 350 seconds upon completion or before the transseptal puncture. Other medications, including $\beta$-blockers, renin-angiotensinaldosterone system (RAAS) antagonists, antiarrhythmic drugs and proton-pump inhibitors (PPI), were also used based on the standard treatment regimen and left to the clinician's discretion.

\section{Follow-ups}

Standard practices, including a 12-lead ECG and 24$\mathrm{h}$ Holter monitoring, were performed during outpatient clinic visits by specialized cardiologists or echocardiography specialists at each visit. ECG and 24-h Holter monitoring in AF patients were mainly suitable to evaluate the adequacy of heart rate control, related symptoms with AF recurrences, and detect focal induction of bouts of paroxysmal AF. Additional transthoracic echocardiography was carried out for identifying thrombosis formation in the left atrial and assessment of left ventricular size and function (systolic and diastolic), atrial size, and right heart function. Laboratory data and clinical features were collected from the medical records, including hemoglobin level, PLT count, coagulation function tests and the incidence of bleeding events. We recorded frequencies of thrombosis and bleeding events during 12-month follow-ups. Incidence of thrombotic and bleeding events was calculated based on frequencies in 12 months.

\section{Clinical Outcomes}

Routine blood tests, coagulation function tests (prothrombin time, activated partial thromboplastin time, fibrinogen, D-dimer value), and concomitant medication were recorded. The main effective endpoint was the recurrence of systemic embolism, including stroke diagnosed by neurology examination based on computed tomography (CT) or magnetic resonance imaging (MRI), pulmonary embolism diagnosed by CT pulmonary angiography (CTPA) or ventilation-perfusion lung scan, venous thromboembolism diagnosed by ultrasound scanning and cardiac embolism diagnosed by echocardiography during 12-month followups. All these events were adjudicated by the Antithrombotic Therapy and Prevention of Thrombosis Guideline. $^{12}$ The principal safety outcome was to compare the rate of major and clinically relevant, nonmajor bleeding complications such as gastrointestinal hemorrhage, hematuria, epistaxis, operation site hemorrhage, and bleeding gums for patients assigned to rivaroxaban or dabigatran. Definitions of major and clinically relevant nonmajor bleeding complications were concordant with the guidance of the International Society on Thrombosis and Haemostasis. $^{13}$ Major bleeding was defined as a reduction in the hemoglobin level of at least $20 \mathrm{~g}$ per liter, transfusion of at least two units of blood, or symptomatic bleeding in a critical organ. Clinically relevant nonmajor bleeding was defined as bleeding requiring medical intervention by a health-care professional, deeper level of care, or onsite evaluation.

The composite endpoint was defined as time from the inclusion to the occurrence of systemic embolism or bleeding complications, whichever occurred first. Events were screened during the period from study inclusion until the completion of data collection over 12 months. Pharmacy care helped to confirm the enrolled patients with continuous and appropriate anticoagulation strategy and monitor adverse drug reaction during the follow-ups. Adherence to 
DOAC therapy was measured by proportion of days covered (PDC), defined as the total number of days covered with a DOAC divided by observational time interval. ${ }^{14}$ All of the patients enrolled in the study claimed they took the medicine on a daily basis. Thus, all of the patients were considered having good adherence to DOAC $(\mathrm{PDC}=100 \%)$.

\section{Statistical Analysis}

The descriptive statistics of continuous variables were expressed as means $\pm \mathrm{SD}$, while those of discrete variables were expressed as counts or percentages. Two-way ANOVA was applied to compare the differences of the continuous variables among the four groups of patients and chi-squared tests were conducted to compare the distribution of categorical variables.

The comparison of thrombosis and bleeding complications were analyzed by carrying out chi-squared test. We adopted the Kaplan-Meier method for survival curve analysis by using the log rank test for trend and the Cox regression analysis. We compared time to thrombosis (defined as time from inclusion to first occurrence of stroke or systemic embolism), time to bleeding (defined as time from inclusion to first occurrence of bleeding) and time to cumulative events (defined as time from inclusion to first occurrence of thrombosis or major and clinically relevant nonmajor bleeding) among the four groups for patients during anticoagulation with either rivaroxaban or dabigatran. Results for Cox regression analysis were presented as HRs along with a two-sided $P$-value. A two-sided $P$ value was used to determine significance (threshold, $P<0.05$ ).

We analyzed BMI categories associations of thrombosis, bleeding complications and composite endpoint by using multiple statistical models. Variables analyzed for thrombosis and bleeding included potential thrombosis and bleeding risk factors (age, gender, BMI, hypertension, diabetes mellitus, stroke, heart failure, coronary artery disease, and peripheral artery disease). Variables analyzed for composite endpoint included BMI, potential bleeding and thrombosis risk factors mentioned above. ORs with two-sided $95 \%$ CIs were calculated for the risk factors of thrombosis, bleeding complications and composite endpoint. Results are presented as ORs along with 95\%CI.

We performed correlation tests to analyze the BMI impact on effectiveness or safety during anticoagulation treatment. Statistical analysis was conducted using SPSS Statistics v. 22 (IBM Corporation, Armonk, NY, USA) and
Prism 5 (GraphPad Software). A $P$-value of 0.05 was considered the threshold for statistical significance.

\section{Results \\ Patient Characteristics}

During the study inclusion period, 2402 NVAF patients who underwent catheter ablation and medicated with DOACs were enrolled in our study (1290 received dabigatran, $53.7 \%$ and 1112 received rivaroxaban, $46.3 \%$ ).

According to WHO classification, 1362 (56.7\%) were nonobese (BMI $\leq 24.9 \mathrm{~kg} / \mathrm{m}^{2}$ ) among the enrolled patients. The rest of patients were obese: 521 (21.7\%) patients were preobese (BMI, $\left.25.0-29.9 \mathrm{~kg} / \mathrm{m}^{2}\right), 344(14.3 \%)$ were class I obese (BMI $30.0-34.9 \mathrm{~kg} / \mathrm{m}^{2}$ ) individuals and 175 $(7.3 \%)$ were class II+ obese (BMI $\geq 35.0 \mathrm{~kg} / \mathrm{m}^{2}$ ) (Table 1).

Four investigated BMI subgroups were not significantly different regarding sex, age, eGFR, laboratory indicators, predetermined stroke risk $\left(\mathrm{CHA}_{2} \mathrm{DS}_{2}-\mathrm{VAS}_{\mathrm{C}}\right.$; $P=0.225$ ). However, the prevalence of risk factors for cardiovascular disease, such as diabetes and related laboratory indicators with hemoglobin A1c, was progressively higher in class II + obese patients, $P<0.05$ (Table 1).

\section{Clinical Outcomes Assessments of Normal Weight and Preobese Patients in Comparison to Obese Patients}

During the follow-up up to 12 months, there was significant difference among the four groups in terms of systemic thromboembolism occurrence ratio, including stroke, pulmonary embolism, venous thromboembolism, and cardiac embolism. The incidence of systemic embolism complications was higher in the obese group (nonobese 1.5\% vs preobese $1.4 \%$ vs class I obese $2.7 \%$ vs class II+ obese $5.7 \%$; $P=0.001$ ) (Table 2). A high incidence of occurrence stroke was discovered in class II + obese group, compared to the preobese and nonobese $(P=0.003)$.

Multivariate logistic regression was performed to identify the independent associations of the thrombosis with BMI and potential risk factors. By multivariate analysis, a higher BMI value (BMI $\left.\geq 25 \mathrm{~kg} / \mathrm{m}^{2}\right) \quad(P=0.019$; $\mathrm{OR}=2.094,95 \% \mathrm{CI}: 1.129-3.883$ ) was an independent predictor for thrombosis in patients treated with dabigatran or rivaroxaban, as shown in Table 3.

In the 12-month follow-up period, $46(1.9 \%)$ patients, including $20(1.5 \%)$ for dabigatran and $26(2.3 \%)$ for rivaroxaban, experienced system thrombotic incident. Cumulative Kaplan-Meier results illustrated that there 
Table I Baseline Characteristics Stratified According to the BMI Categories

\begin{tabular}{|c|c|c|c|c|c|}
\hline Baseline Characteristics & $\begin{array}{l}\text { BMI }<25 \\
(n=1362)\end{array}$ & $\begin{array}{c}\leq 25 \text { BMI<30 } \\
(n=52 I)\end{array}$ & $\begin{array}{c}\leq 30 \text { BMI }<35 \\
(n=344)\end{array}$ & $\begin{array}{l}B M I \geq 35 \\
(n=I 75)\end{array}$ & $P$-value \\
\hline Rivaroxaban, n (\%) & $632(46.4 \%)$ & $250(48.0 \%)$ & $153(44.5 \%)$ & 77 (44.0\%) & \multirow[t]{2}{*}{0.698} \\
\hline Dabigatran, n (\%) & $730(53.6 \%)$ & $271(52.0 \%)$ & 191 (55.5\%) & 98 (56.0\%) & \\
\hline Age, years; mean (SD) & $66.1(12.4)$ & $67.6(13.1)$ & $67.0(12.7)$ & $65.5(11.2)$ & 0.094 \\
\hline Gender, male, n (\%) & $787(57.8 \%)$ & $317(60.8 \%)$ & 198 (57.6\%) & $116(66.3 \%)$ & 0.127 \\
\hline Smoking, n (\%) & $243(17.8 \%)$ & $100(19.2 \%)$ & 66 (19.2\%) & $4 \mathrm{I}(23.4 \%)$ & 0.339 \\
\hline Alcohol, n (\%) & $167(12.3 \%)$ & $68(13.1 \%)$ & 37 (10.8\%) & 32 (18.3\%) & 0.093 \\
\hline \multicolumn{6}{|l|}{ Comorbidity } \\
\hline Hypertension, n (\%) & 995 (73.1\%) & $412(79.1 \%)$ & $260(75.6 \%)$ & 132 (75.4\%) & 0.060 \\
\hline Dyslipidemia, n (\%) & $225(16.5 \%)$ & 105 (20.2\%) & 76 (22.1\%) & 35 (20.0\%) & 0.052 \\
\hline Diabetes, n (\%) & $200(14.7 \%)$ & $102(19.6 \%)$ & $108(31.4 \%)$ & $70(40.0 \%)$ & $0.000 *$ \\
\hline CKD, n (\%) & $177(13.0 \%)$ & 71 (13.6\%) & 48 (I4.0\%) & 30 (17.1\%) & 0.506 \\
\hline Stroke/TIA, n (\%) & $211(15.5 \%)$ & $96(18.4 \%)$ & $58(16.9 \%)$ & $30(17.1 \%)$ & 0.476 \\
\hline Liver disease, n (\%) & 73 (5.4\%) & $28(5.4 \%)$ & $14(4.1 \%)$ & II (6.3\%) & 0.708 \\
\hline Heart failure, $\mathrm{n}(\%)$ & $190(14.0 \%)$ & $67(12.9 \%)$ & 49 (I4.2\%) & $23(13.1 \%)$ & 0.916 \\
\hline PAD, n (\%) & $73(5.4 \%)$ & $23(4.4 \%)$ & $18(5.2 \%)$ & $8(4.6 \%)$ & 0.847 \\
\hline CAD, n (\%) & $307(22.5 \%)$ & $116(22.3 \%)$ & $78(22.7 \%)$ & $33(18.9 \%)$ & 0.737 \\
\hline \multicolumn{6}{|l|}{ Laboratory tests } \\
\hline eGFR, $\mathrm{mL} /\left(\min \cdot 1.73 \mathrm{~m}^{2}\right) ;$ mean (SD) & $77.6(20.9)$ & $79.4(33.6)$ & $75.7(18.4)$ & $76.4(17.4)$ & 0.128 \\
\hline $\mathrm{Hb}, \mathrm{g} / \mathrm{L} ;$ mean (SD) & $135.2(17.3)$ & $135.2(17.1)$ & $135.9(16.9)$ & $138.2(15.3)$ & 0.173 \\
\hline $\mathrm{PLT}, * 10^{9} / \mathrm{L} ;$ mean $(\mathrm{SD})$ & $194.5(57.0)$ & 195.1 (59.4) & $191.9(55.4)$ & $189.5(55.9)$ & 0.613 \\
\hline ALT, U/L; mean (SD) & $26.8(52.3)$ & $25.2(21.0)$ & $27.3(54.6)$ & $30.0(52.1)$ & 0.700 \\
\hline Hemoglobin Alc, \%; mean (SD) & $6.0(1.4)$ & $6.1(1.3)$ & $6.1(1.4)$ & $6.3(1.3)$ & $0.020 *$ \\
\hline APTT, s; mean (SD) & $30.7(6.0)$ & $30.5(5.7)$ & $31.4(17.6)$ & $31.1(6.4)$ & 0.444 \\
\hline PT, s; mean (SD) & I3.I (4.0) & I3.3 (3.8) & $13.6(4.5)$ & $13.6(4.2)$ & 0.215 \\
\hline TT, s; mean (SD) & $29.0(32.1)$ & $29.4(32.81)$ & $28.7(32.4)$ & $29.7(32.8)$ & 0.978 \\
\hline D-Dimer, mg/L; mean (SD) & $0.71(1.5)$ & $0.78(1.7)$ & $0.80(1.6)$ & $0.78(2.3)$ & 0.764 \\
\hline \multicolumn{6}{|l|}{ Co-medication } \\
\hline Statin, n (\%) & $499(36.6 \%)$ & $166(31.9 \%)$ & $114(33.1 \%)$ & $55(31.4 \%)$ & 0.151 \\
\hline Anti-hypertension, n (\%) & 994 (73.0\%) & $394(75.6 \%)$ & $250(72.7 \%)$ & $126(72 \%)$ & 0.635 \\
\hline$\beta$-blockers, n (\%) & 469 (34.4\%) & $176(33.8 \%)$ & 117 (34.0\%) & $60(34.3 \%)$ & 0.994 \\
\hline Antiplatelet, n (\%) & $287(21.1 \%)$ & 117 (22.5\%) & $76(22.1 \%)$ & $50(28.6 \%)$ & 0.159 \\
\hline Anti-arrythmic agent, $\mathrm{n}(\%)$ & $300(22.0 \%)$ & $109(20.9 \%)$ & $78(22.7 \%)$ & $4 \mathrm{I}(23.4 \%)$ & 0.884 \\
\hline PPI, n (\%) & $46 \mathrm{I}(33.8 \%)$ & $193(37.0 \%)$ & $112(32.6 \%)$ & $56(32.0 \%)$ & 0.434 \\
\hline $\mathrm{CHA}_{2} \mathrm{DS}_{2}$-VASc; mean (SD) & $2.8(1.5)$ & $2.9(1.6)$ & $3.0(1.6)$ & $2.9(1.5)$ & 0.225 \\
\hline $\mathrm{CHA}_{2} \mathrm{DS}_{2}$-VASc groups of 2 or less; $n(\%)$ & $620(45.5 \%)$ & $245(47.0 \%)$ & $134(39.0 \%)$ & $80(45.7 \%)$ & 0.106 \\
\hline $\mathrm{CHA}_{2} \mathrm{DS}_{2}$-VASc groups of $3 ; \mathrm{n}(\%)$ & $314(23.1 \%)$ & $103(19.8 \%)$ & $83(24.1 \%)$ & $44(25.1 \%)$ & 0.303 \\
\hline $\begin{array}{l}\mathrm{CHA}_{2} \mathrm{DS}_{2} \text {-VASc groups of } 4 \text { or more; } \\
n(\%)\end{array}$ & $428(31.4 \%)$ & $173(33.2 \%)$ & $127(36.9 \%)$ & $51(29.1 \%)$ & 0.194 \\
\hline HAS-BLED; mean (SD) & $2.0(1.0)$ & $2.2(1.08)$ & $2.0(1.1)$ & $2.1(1.3)$ & 0.146 \\
\hline HAS-BLED $\geq 3 ;$ n (\%) & 429 (31.5\%) & $189(36.3 \%)$ & $116(33.7 \%)$ & 68 (38.9\%) & 0.089 \\
\hline
\end{tabular}

Notes: The data is displayed as mean (SD) or percent. P-value was calculated with two-way ANOVA analysis and represented with interaction, ${ }^{*}<0.05$.

Abbreviations: BMI, body mass index; CKD, chronic kidney disease, is defined as eGFR $<60 \mathrm{~mL} /\left(\min \cdot 1.73 \mathrm{~m}^{2}\right)$; TIA, transient ischemic attack; PAD, peripheral artery disease; CAD, coronary artery disease; eGFR, estimated glomerular filtration rate; LDL, low-density lipoprotein; Hct, hematocrit; Hb, hemoglobin; PLT, platelet; ALT, alanine aminotransferase; CK-MB, creatine kinase-MB; NT-proBNP, N-terminal pronatriuretic peptide; APTT, activated partial thromboplastin time; PT, prothrombin time; TT, thrombin time; PPI, proton-pump inhibitor. 
Table 2 Clinical Outcomes Comparison Among the Four Groups

\begin{tabular}{|c|c|c|c|c|c|}
\hline & BMI <25 $(n=1362)$ & $\leq 25 \mathrm{BMI}<30(n=521)$ & $\leq 30$ BMI $<35(n=344)$ & BMI $\geq 35(n=\mid 75)$ & $P$-value \\
\hline Systemic thrombolis, (\%) & 1.5 & 1.4 & 2.7 & 5.7 & $0.001 *$ \\
\hline VTE, (\%) & 0.4 & 0.4 & 0.6 & 1.7 & 0.126 \\
\hline $\mathrm{PE},(\%)$ & 0.7 & 0.6 & 0.9 & 0.6 & 0.949 \\
\hline Stroke, (\%) & 0.2 & 0.0 & 0.6 & 2.3 & $0.003^{*}$ \\
\hline Cardiac thrombosis, (\%) & 0.2 & 0.4 & 0.6 & 1.1 & 0.155 \\
\hline
\end{tabular}

Notes: $* P<0.05$; $P$-value represented with interaction. Systemic thromboembolism is defined as pulmonary embolism, venous thromboembolism, stroke or cardiac embolism. Cardiac thrombosis refers to new occurrence of acute coronary syndrome or left atrium thrombosis.

Abbreviations: VTE, venous thromboembolism; PE, pulmonary embolism.

Table 3 Association of the BMI and Potential Risk Factors with Thrombosis in Patients Treated with Dabigatran or Rivaroxaban

\begin{tabular}{|l|c|c|c|c|}
\hline Variables & SE & P-value & OR & 95\%Cl \\
\hline Gender (male) & 0.323 & 0.294 & 1.405 & $0.746-2.647$ \\
\hline Age $\geq 65$ years & 0.335 & $0.78 I$ & 1.097 & $0.569-2.115$ \\
\hline BMI $\geq 25 \mathrm{~kg} / \mathrm{m}^{2}$ & 0.315 & $0.019 *$ & 2.094 & $1.129-3.883$ \\
HTN & 0.398 & 0.391 & 1.406 & $0.645-3.067$ \\
DM & 0.357 & 0.776 & 1.107 & $0.550-2.228$ \\
HF & 0.420 & 0.753 & 1.142 & $0.50 \mathrm{I}-2.60 \mathrm{I}$ \\
CAD & 0.384 & 0.626 & 0.829 & $0.391-1.760$ \\
Stroke & 0.358 & 0.157 & 1.659 & $0.822-3.348$ \\
PAD & 0.553 & 0.335 & 1.704 & $0.576-5.038$ \\
\hline
\end{tabular}

Note: $* p<0.05$.

Abbreviations: BMI, body mass index; HTN, hypertension; DM, diabetes mellitus; HF, heart failure; CAD, coronary artery disease; PAD, peripheral artery disease.

was no significant difference in the incidence of the primary effectiveness endpoint of systemic thrombosis during the medication with dabigatran among the four group

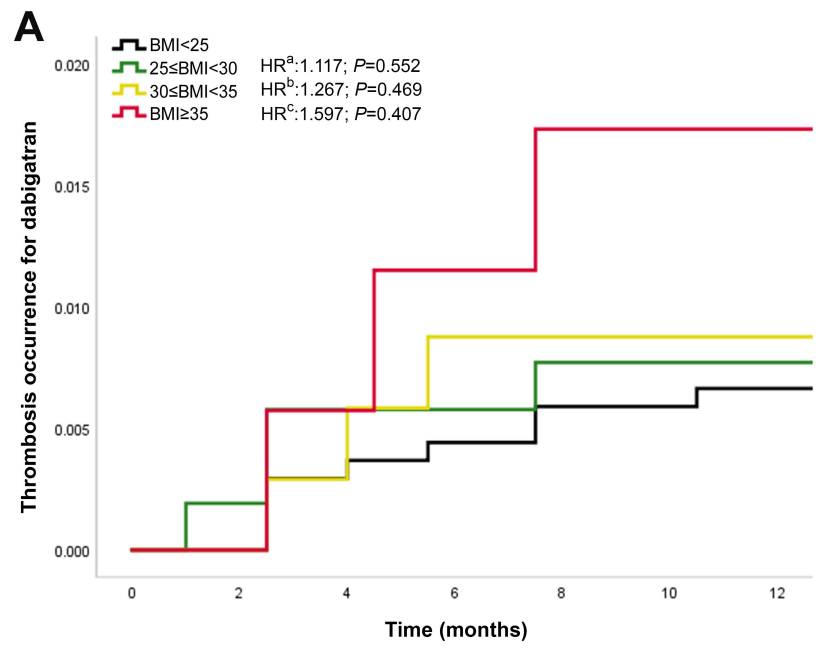

patients. In the whole cohort of rivaroxaban treated patients, those who belong to higher BMI subgroups were more likely to experience shorter time to thrombosis 12-month TTT rates of $0.5 \%$ for nonobese vs $1.7 \%$ for class I obese patients, $\mathrm{HR}=3.716, P=0.005 ; 12$-month TTT rates of $0.5 \%$, for nonobese vs $4.0 \%$ for class II+ obese patients, $\quad \mathrm{HR}=6.843, \quad P=0.001)$, as demonstrated in Figure 1.

\section{Anticoagulation-related Complications}

The frequencies of bleeding events in the nonobese and obese groups are shown in Table 4 . With regard to gastrointestinal hemorrhage, no significant difference was found among the four groups (nonobese $3.30 \%$ vs preobese $3.26 \%$ vs class I obese $2.91 \%$ vs class II+ obese $1.71 \%$, $P=0.710$ ). The cumulative incidence of bleeding complications such operation site hemorrhage, skin ecchymosis and bleeding gums during anticoagulation therapy were similar among the four groups $(P>0.05)$. There was no significant

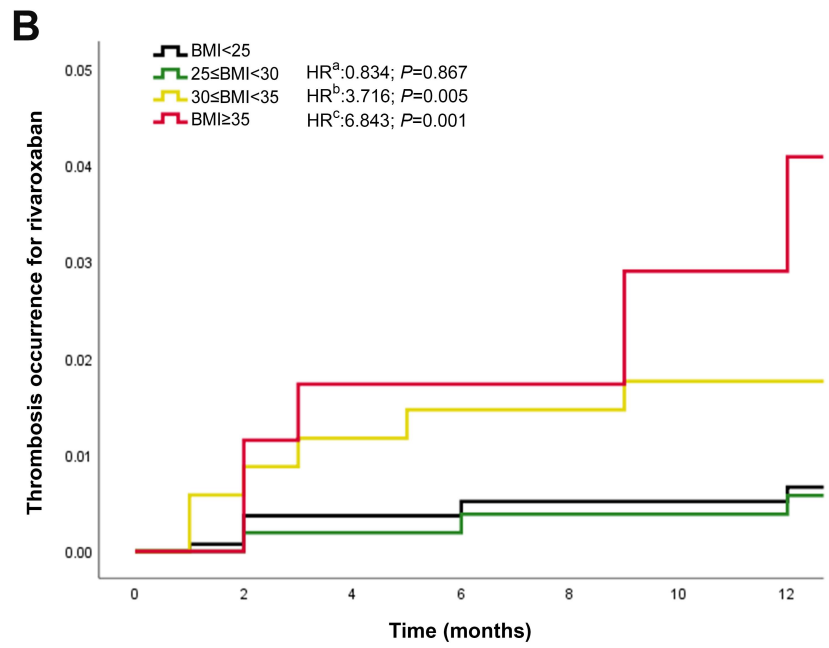

Figure I Time to thrombosis (TTT) in dabigatran (A) and rivaroxaban (B) treated patients, stratified into four subgroups (nonobesity, preobese, class I and class II+ obesity) according to the body mass index (BMI). ${ }^{a}$ Referred as the comparison between preobese and nonobesity. ${ }^{b}$ Referred as the comparison between class I obesity and nonobesity. ${ }^{\mathrm{C}}$ Referred as the comparison between class II+ obesity and nonobesity. 
Table 4 Bleeding Complications Comparison Within 12-month Follow-ups

\begin{tabular}{|l|c|c|c|c|c|}
\hline Bleeding Events & $\begin{array}{l}\text { BMI } \\
<\mathbf{2 5}\end{array}$ & $\begin{array}{l}\leq \mathbf{2 5} \\
\text { BMI } \\
<30\end{array}$ & $\begin{array}{l}\leq \mathbf{3 0} \\
\text { BMI } \\
<35\end{array}$ & $\begin{array}{l}\text { BMI } \\
\geq 35\end{array}$ & P-value \\
\hline $\begin{array}{l}\text { Gastrointestinal } \\
\text { hemorrhage, (\%) }\end{array}$ & 3.30 & 3.26 & 2.91 & 1.71 & 0.710 \\
\hline Hematuria & 1.2 & 1.34 & 0.58 & 0.57 & 0.732 \\
\hline Epistaxis (\%) & 0.3 & 0.19 & 0.58 & 0.00 & 0.683 \\
\hline $\begin{array}{l}\text { Operation site } \\
\text { hemorrhage (\%) }\end{array}$ & 1.3 & 1.15 & 1.45 & 0.00 & 0.523 \\
\hline Bleeding gums (\%) & 0.9 & 0.77 & 0.87 & 0.57 & 1.000 \\
\hline Skin ecchymosis (\%) & 0.9 & 1.15 & 0.58 & 0.57 & 0.844 \\
\hline PLT <125*10\%/L (\%) & 11.6 & 8.64 & 10.76 & 12.57 & 0.219 \\
\hline $\begin{array}{l}\text { Male: } \mathrm{Hb}<120 \mathrm{~g} / \mathrm{L}(\%) \\
\text { Female: } \mathrm{Hb}<110 \mathrm{~g} / \mathrm{L}(\%)\end{array}$ & 11.0 & 9.98 & 7.56 & 6.29 & 0.088 \\
\hline PT >13s (\%) & 22.7 & 23.03 & 24.13 & 22.86 & 0.955 \\
\hline
\end{tabular}

Abbreviations: PLT, platelet; Hb, hemoglobin; PT, prothrombin time. P-value represented with interaction.

difference between the nonobese and obese groups with respect to the levels of $\mathrm{Hb}$, PLT, and PT under the coagulation threshold $(P>0.05)$.

Considering the whole cohort of patients, no significant difference was observed in terms of the time to bleeding occurrence among the four group patients treated either with rivaroxaban or dabigatran (Figure 2).

Multivariate logistic regression was performed to identify the independent associations of bleeding complications
Table 5 Association of the BMI and Potential Risk Factors with Bleeding Complications in Patients Treated with Dabigatran or Rivaroxaban

\begin{tabular}{|l|c|c|c|c|}
\hline Variables & SE & P-value & OR & $95 \% \mathbf{C l}$ \\
\hline Age $\geq 65$ years & 0.214 & $0.08 \mathrm{I}$ & $\mathrm{I} .726$ & $\mathrm{I} .134-2.628$ \\
\hline BMI $\geq 25 \mathrm{~kg} / \mathrm{m}^{2}$ & 0.187 & 0.434 & 0.864 & $0.599-1.246$ \\
Alcohol & 0.272 & 0.484 & 1.210 & $0.709-2.064$ \\
HTN & 0.209 & 0.480 & 0.863 & $0.573-1.300$ \\
CKD & 0.240 & 0.204 & 1.356 & $0.847-2.17 \mathrm{I}$ \\
\hline Liver dysfunction & 0.593 & 0.191 & $0.46 \mathrm{I}$ & $0.144-1.473$ \\
Stroke & 0.235 & 0.623 & 1.122 & $0.708-1.779$ \\
Antiplatelet & 0.221 & $0.88 \mathrm{I}$ & 1.034 & $0.670-1.594$ \\
\hline
\end{tabular}

Abbreviations: BMI, body mass index; HTN, hypertension; CKD, chronic kidney disease $\left(\mathrm{eGFR}<60 \mathrm{~mL} / \mathrm{min} \cdot 1.73 \mathrm{~m}^{2}\right.$ ).

with BMI and potential bleeding risk factors. By multivariate analysis, no risk factor was found as an independent predictor for bleeding complications in patients treated with dabigatran or rivaroxaban, as shown in Table 5.

\section{Composite Endpoint Analysis of Nonobese Patients in Comparison to Obese Patients}

There were 170 (7.7\%) patients who experienced an event with either thrombosis and bleeding for patients receiving rivaroxaban or dabigatran, and we observed no substantial differences in the results of the composite endpoints among the four groups (overall $P=0.967$, with 12-month composite endpoint rates of $6.7 \%, 6.7 \%, 7.3 \%$, and $7.4 \%$ for nonobese, preobese, class I and class II+ obese patients, respectively).
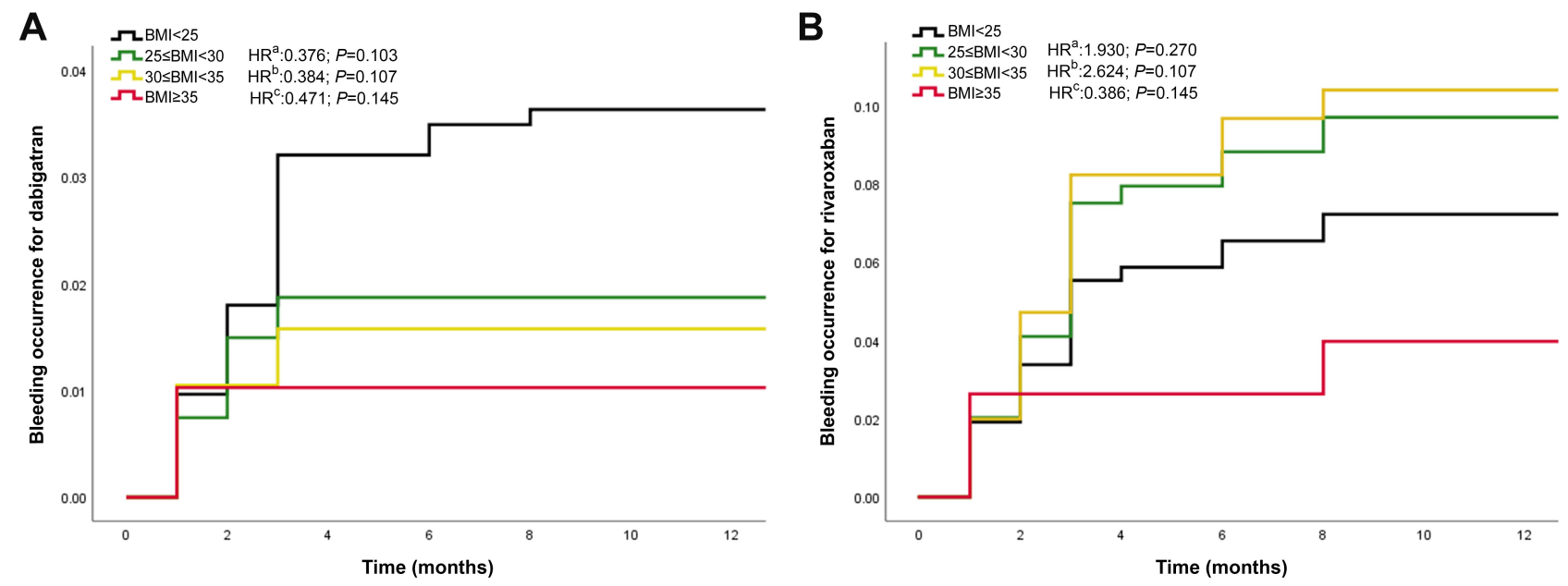

Figure 2 Time to bleeding (TTB) in dabigatran (A) and rivaroxaban (B) treated patients, stratified into four subgroups (nonobesity, preobese, class I and class II+ obesity) according to the body mass index (BMI). ${ }^{a}$ Referred as the comparison between preobese and nonobesity. ${ }^{b}$ Referred as the comparison between class I obesity and nonobesity. ${ }^{\mathrm{C}}$ Referred as the comparison between class II+ obesity and nonobesity. 

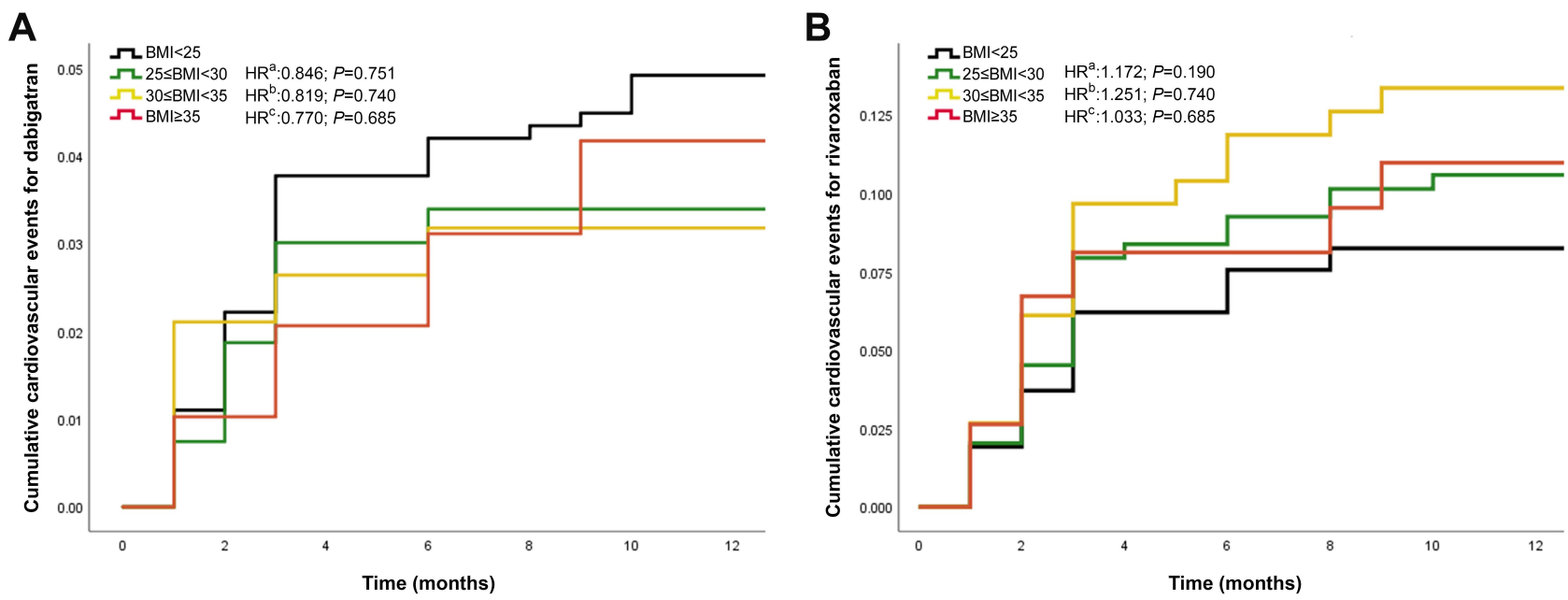

Figure 3 Cumulative events curves in dabigatran (A) and rivaroxaban (B) treated patients, stratified into four subgroups (nonobese, preobese, class I and class II+ obese) according to the BMI. ${ }^{\text {a }}$ Referred as the comparison between preobese and nonobesity. ${ }^{b}$ Referred as the comparison between class I obesity and nonobesity. ${ }^{\mathrm{C}}$ Referred as the comparison between class II+ obesity and nonobesity.

We further performed the same analysis to compare time to cumulative events among the four groups for rivaroxaban and dabigatran. There was no statistically significant difference in terms of the time to cumulative events among the four groups of patients treated both with rivaroxaban and with dabigatran (Figure 3).

Multivariate logistic regression was performed to identify the independent associations of the composite endpoints with BMI, potential thrombosis and bleeding risk factors. By multivariate analysis, no risk factor was found as an independent predictor for composite endpoint in patients treated with NOACs, as shown in Table 6.

The linear regression with BMI and clinical outcomes was calculated to evaluate the relationship among them (Figure 4). Briefly, the thrombosis and bleeding rate increased with the increase in BMI levels. A positive linear relationship was observed between BMI levels and occurrence rate of thrombosis and bleeding in under anticoagulation patients with NVAF $\left(\mathrm{R}^{2}=0.451\right.$ and $\mathrm{R}^{2}=0.383$, respectively).

\section{Discussion}

To the best of our knowledge, our study is the first to show statistically increased risks for thrombotic incidents in Chinese obese patients with atrial fibrillation anticoagulated with low dose DOACs. Using DOACs might be alternatives for the treatment of patients with high BMI referred to catheter ablation of atrial fibrillation. We observed a comparable cardiovascular safety for patients with high BMI in this large setting of catheter ablation for real-life DOAC recipients. Meanwhile, rivaroxaban dose increase was suggested for obese patients.

The predictable anticoagulant response of DOACs has provided the pharmacological basis for their administration in fixed doses without routine coagulation monitoring. Although it is possible to accurately measure DOAC drug levels, within patient variability complicates interpretation of these results. Furthermore, patient characteristics

Table 6 Association of the BMI, Potential Thrombosis and Bleeding Risk Factors with Composite Endpoint in Patients Treated with Dabigatran or Rivaroxaban

\begin{tabular}{|l|c|c|c|c|}
\hline Variables & SE & Pvalue & OR & $95 \% \mathrm{CI}$ \\
\hline Gender (male) & 0.181 & 0.867 & 0.970 & $0.680-1.384$ \\
\hline Age $\geq 65$ years & 0.193 & 0.107 & 1.490 & $1.024-2.179$ \\
\hline BMI $\geq 25 \mathrm{~kg} / \mathrm{m}^{2}$ & 0.165 & 0.207 & 1.230 & $0.89 I-1.704$ \\
Smoking & 0.264 & 0.587 & 1.150 & $0.688-1.935$ \\
Alcohol & 0.305 & 0.484 & 1.238 & $0.68 \mathrm{I}-2.252$ \\
HTN & 0.192 & 0.895 & 0.975 & $0.669-1.421$ \\
DM & 0.213 & 0.394 & 0.834 & $0.549-1.267$ \\
CKD & 0.219 & 0.145 & 1.376 & $0.896-2.113$ \\
\hline Liver dysfunction & 0.520 & 0.146 & 0.470 & $0.169-1.302$ \\
HF & 0.234 & 0.770 & 1.071 & $0.677-1.693$ \\
CAD & 0.189 & 0.232 & 1.254 & $0.965-1.817$ \\
Stroke & 0.210 & 0.454 & 1.170 & $0.776-1.765$ \\
PAD & 0.307 & 0.131 & 1.590 & $0.87 I-2.903$ \\
Antiplatelet & 0.195 & 0.468 & 1.152 & $0.786-1.687$ \\
\hline
\end{tabular}

Abbreviations: BMI, body mass index; HTN, hypertension; DM, diabetes mellitus; CKD, chronic kidney disease (eGFR $<60 \mathrm{~mL} / \mathrm{min} \cdot 1.73 \mathrm{~m}^{2}$ ); $\mathrm{HF}$, heart failure; CAD, coronary artery disease; PAD, peripheral artery disease. 

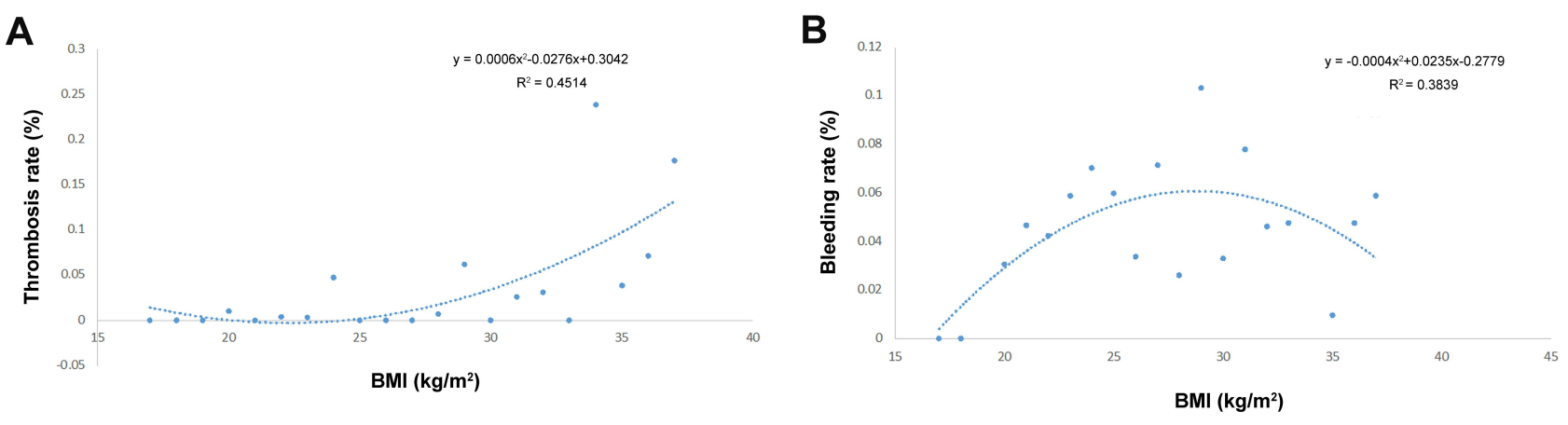

Figure 4 (A) Linear regression of $B M I$ levels and thrombosis occurrence rate $\left(R^{2}=0.45 I\right)$. $(B)$ Linear regression of $B M I$ levels and bleeding occurrence rate $\left(R^{2}=0.383\right)$.

confound the association between DOAC drug levels and clinical outcomes and previous studies revealed that coagulation pharmacodynamic markers were suggested for obese patients. ${ }^{15}$ Past conclusions regarding relationship between BMI and clinical outcomes in patients treated with DOACs had been controversial. ${ }^{16}$ Some real-word studies suggested no indication that elevated BMI is associated with a lack of DOAC effectiveness or safety, ${ }^{17-19}$ while one retrospective study indicated that patients with higher BMI treated with DOAC experienced worse clinical outcomes. ${ }^{6}$ Our results showed partly similar clinical outcomes with the retrospective study, suggesting that patients with higher BMI could be significantly prone to encounter thrombotic incidents during anticoagulation with lower dose DOACs, especially for stroke events. ${ }^{6}$ Metabolic disturbances may play an important role in the development of stroke, and moreover, the duration of diabetes mellitus was a significant cofactor, representing a dose-response relationship of diabetes mellitus exposure to stroke severity. ${ }^{20}$

Our results were based on a real cohort study of nonvalvular atrial fibrillation patients under low dose DOAC anticoagulation with 12-month follow-ups. We evaluated the thrombosis and bleeding rate for dabigatran and rivaroxaban for 12-month follow-ups. The observation for the recurrent thrombosis rate of dabigatran in our study was similar to that reported in a previous study within the range of $1.8 \%{ }^{21}$ Our results revealed that patients with a higher BMI were associated with higher thrombosis risk. Theoretically, the distribution volume of drugs can increase with increasing body weight or BMI, which would result in a hypothetical risk for relative underdosing. The previous study showed that lower body weight was associated with $21 \%$ higher concentration of dabigatran when compared with patients with a normal body weight, and obese patients (weight $>100 \mathrm{~kg}$ ) had 53\% lower level of dabigatran than patients with a normal body weight. ${ }^{22}$ Our results showed that rivaroxaban anticoagulated patients with BMI $>30 \mathrm{~kg} / \mathrm{m}^{2}$ tended to experience a high rate of thrombosis occurrence that might worsen clinical outcomes. The lower antithrombotic effectiveness may occur due to the dilutional effect of a higher distribution volume. ${ }^{23}$ Recent data implied that patients with higher BMI and receiving factor Xa inhibitors were more likely to experience stroke/systemic embolism sooner. $^{6}$

Our results indicated that the trend of DOAC-related bleeding events was relatively low in the extreme weight group (BMI $\geq 35 \mathrm{~kg} / \mathrm{m}^{2}$ ), although there is no significant difference across the whole cohort. A previous study observed BMI $<30 \mathrm{~kg} / \mathrm{m}^{2}$ was associated with the highest occurrence rate of bleeding events, and bleeding occurrence decreased as the BMI groups increased in the treatment of DOAC. ${ }^{17}$ A meta-analysis suggested the comparable safety and effectiveness of DOACs, compared with vitamin $\mathrm{K}$ antagonists in patients stratified to different BMI. ${ }^{24}$

In terms of dabigatran treated group, a lower BMI was more associated with bleeding events sooner. Our finding was consistent with a recent study, which might be explained by pharmacokinetics. ${ }^{25}$ In RE-LY trial, dabigatran trough concentration was inversely proportional to body weight and bleeding outcomes were correlated with dabigatran plasma concentrations. ${ }^{4}$ With regards to liposolubility, DOACs blood concentration might fluctuate due to volumes of distribution. ${ }^{26}$ Considering rivaroxaban, a factor Xa inhibitor, our data displayed that patients with $\mathrm{BMI} \geq 35 \mathrm{~kg} / \mathrm{m}^{2}$ were less prone to experience bleeding events due to low dose regimen contributing to lower drug exposure, but sooner than other groups. Lucijanic et al came to an opposite conclusion that this population has a higher risk of bleeding. ${ }^{6}$ The reason still remains 
unclear because the effects of body weight on the pharmacokinetics and pharmacodynamics of rivaroxaban were considered small. ${ }^{27-29}$

The difference in results may not only be caused by different pharmacological mechanisms. Interestingly, in the study of edoxaban, another factor Xa inhibitor, the conclusion was consistent with our dabigatran study. ${ }^{30}$ A review by Covert et al recommended that edoxaban and dabigatran should be avoided in patients at morbid obesity. ${ }^{31}$

Although our results suggested no influence of BMI on safety outcomes with anticoagulation-related bleeding complication, there seemed to be a high bleeding trend on bleeding site of gastrointestinal, hematuria, epidural hematoma, and skin ecchymosis in preobese and nonobese groups, compared with class I and class II+ obese individuals which might be explained by the low dose regimen. Recent data from a meta-analysis implied that there is no statistically significant difference in bleeding complications regarding different BMI subgroups in patients treated with DOAC, which is inconsistent with our findings. ${ }^{32}$ Higher BMI subgroups were more likely to experience bleeding sooner during medication with rivaroxaban. It might be explained that physicians now realized the potential bleeding risks for lower BMI patients and would take protective measures to reduce the risk of bleeding. ${ }^{33}$

Similar to the retrospective study that suggested that event-free outcomes for patients with higher BMI was significantly shortened, ${ }^{6}$ our results displayed a trend that patients with higher BMI in both dabigatran and rivaroxaban groups might experience thrombosis or bleeding events sooner. As bleeding accounted for the majority of cardiovascular events, and difference on anticoagulantrelated bleeding events was not evident among various groups. Moreover, it should be noted that physicians might take precautionary measures to prevent bleeding for the elderly and renal insufficiency such as PPIs for gastrointestinal bleeding which might affect statistical analysis on the incidence of bleeding among patients with diverse obesity level. ${ }^{34,35}$ It was important to mention that controversial findings across other studies might be due to several issues, such as differing outcome definitions, patient cohorts with different risk factor distributions, or different DOACs included.

Our results showed that the occurrence rate of overt thrombosis events increased as the BMI group increased. One alternative viewpoint of this identified trend may be that patients with an elevated BMI may have altered pharmacokinetics of the DOACs resulting in the higher occurrence rate of thrombosis. ${ }^{36}$ Enlarged number and size of adipocytes in obese humans causes systemic microinflammation, which in the long-term reduces the endothelial barrier function when compared with the nonobese population and leads to occurrence of thrombosis events. ${ }^{37}$ Our findings indicated that with the BMI groups range from 25 to $30 \mathrm{~kg} / \mathrm{m}^{2}$ were associated with the highest occurrence rate of bleeding. For patients with BMI $>30 \mathrm{~kg} / \mathrm{m}^{2}$, bleeding rate decreased with increasing BMI due to lower drug exposure. Meanwhile, bleeding rate increased for groups with BMI $<25 \mathrm{~kg} / \mathrm{m}^{2}$. The probable explanation might be that high occurrence of cardiovascular disease such as hypertension, hyperlipidemia and diabetes along with the increasing BMI contributed to the damage of microvascular endothelial structure. ${ }^{38}$

In 2016, the ISTH published guidance for the use of the DOACs in obese patients. ${ }^{39}$ The authors indicated that the statement was meant to provide practical guidance to clinicians and highlights the limited evidence for the use of DOACs in obese patients. In this regard, it suggested that DOACs not be used in patients who meet these high BMI criteria because of limited clinical and pharmacokinetics data, which suggest that decreased drug concentrations and subsequent underdosing may be seen in patients at extremes of weight, which might worsen clinical outcomes. When interpreting our data, we found that use of DOACs appears to have considerable safety in obese patients. Meanwhile, for Chinese patients with higher BMI (BMI $\geq 25 \mathrm{~kg} / \mathrm{m}^{2}$ ), rivaroxaban of lower dosage (15 mg) might be not enough to prevent thrombosis and a higher dose was suggested. DOACs might be considered a possible option in obese patients.

We acknowledge several limitations in our work. First, owing to the nature of this retrospective study, the design carried inherent biases. Second, we evaluated the impacts of BMI on clinical outcomes across different DOACs and in a pooled analysis of thrombosis and bleeding associated conditions that carry different acute or chronic thromboembolic risks and usually affect patients with different characteristics. Third, we conducted a limited follow-up period of 12 months and our sample population was rather small, the power to detect the associations of BMI with outcomes was limited, and the possibility of type II error (ie, a false-negative association) was important to consider. Thus, the long-term BMI impacts might influence clinical outcomes. Fourth, we performed this study with lower dose regime, not the standard doses of DOACs 
which might be a major limitation. Fifth, we did not set VKA as parallel group, which might not reflect the BMI influence on the clinical outcomes for nonvalvular atrial fibrillation patients treated with anticoagulants. Sixth, we did not assess plasma dabigatran or rivaroxaban concentrations and consequently miss pharmacokinetic data that could directly predict the clinical outcomes, and drug interactions with DOACs was not identified. Finally, we acknowledged that the subjective way to measure adherence to DOAC might affect the accuracy of results.

\section{Conclusions}

Despite these limitations, our clinical registry data added to our understandings of the clinical course of obese patients since we displayed difference on effectiveness and safety of DOACs (rivaroxaban and dabigatran) in patients with various BMI categories in the setting of catheter ablation. We found indication that the fixed dose of $15 \mathrm{mg}$ rivaroxaban carried a risk of underexposure, which would lead to an increase of thromboembolic complications in patients with high BMI. Therefore, rivaroxaban dose increase was suggested for obese patients. The use of DOACs appears to have considerable safety in obese patients.

\section{Ethics Statement}

This study was conducted in compliance with the Declaration of Helsinki and Good Clinical Practice in review and was approved by the Ethics Committee of Zhongshan Hospital.

\section{Author Contributions}

All authors made a significant contribution to the work reported, whether that is in the conception, study design, execution, acquisition of data, analysis and interpretation, or in all these areas; took part in drafting, revising or critically reviewing the article; gave final approval of the version to be published; have agreed on the journal to which the article has been submitted; and agree to be accountable for all aspects of the work.

\section{Funding}

This study was supported by the Project of Key Innovative Team of Shanghai Top-Level University Capacity Building in Clinical Pharmacy and Regulatory Science at Shanghai Medical School of Fudan University (No. HJWR-2019-66-19) and Shanghai "Rising Stars of Medical Talent" Youth Development Program-Youth Medical
Talents - Clinical Pharmacist Program (SHWJRS (2019) -072).

\section{Disclosure}

Xiaoye Li and Chengchun Zuo should be regarded as cofirst authors. The authors declare that they have no conflicts of interest in this work.

\section{References}

1. Kirchhof P, Benussi S, Kotecha D, et al.; ESC Scientific Document Group. 2016 ESC guidelines for the management of atrial fibrillation developed in collaboration with EACTS. Eur Heart J. 2016;37 (38):2893-2962.

2. January CT, Wann LS, Calkins H, et al. 2019 AHA/ACC/HRS focused update of the 2014 AHA/ACC/HRS guideline for the management of patients with atrial fibrillation: a report of the American College Of Cardiology/American Heart Association task force on clinical practice guidelines and the heart rhythm society in collaboration with the Society Of Thoracic Surgeons. Circulation. 2019;140 (2):e125-e151. doi:10.1161/CIR.0000000000000665

3. Moore KT, Kröll D. Influences of Obesity and bariatric surgery on the clinical and pharmacologic profile of rivaroxaban. Am J Med. 2017;130(9):1024-1032. doi:10.1016/j.amjmed.2017.05.011

4. Reilly PA, Lehr T, Haertter S, et al. The effect of dabigatran plasma concentrations and patient characteristics on the frequency of ischemic stroke and major bleeding in atrial fibrillation patients: the RE-LY trial (Randomized Evaluation of Long-Term Anticoagulation Therapy). J Am Coll Cardiol. 2014;63(4):321-328. doi:10.1016/j. jacc.2013.07.104

5. Breuer L, Ringwald J, Schwab S, Köhrmann M. Ischemic stroke in an obese patient receiving dabigatran. $N$ Engl J Med. 2013;368 (25):2440-2442. doi:10.1056/NEJMc1215900

6. Lucijanic M, Jurin I, Jurin H, et al. Patients with higher body mass index treated with In review direct/novel oral anticoagulants (DOAC/ DOAC) for atrial fibrillation experience worse clinical outcomes. Int J Cardiol. 2020;301:90-95. doi:10.1016/j.ijcard.2019.10.035

7. Safouris A, Demulder A, Triantafyllou N, Tsivgoulis G. Rivaroxaban presents a better pharmacokinetic profile than dabigatran in an obese non-diabetic stroke patient. J Neurol Sci. 2014;346(1-2):366-367. doi:10.1016/j.jns.2014.09.008

8. Sandhu RK, Ezekowitz J, Andersson U, et al. The 'obesity paradox' in atrial fibrillation: observations from the ARISTOTLE (Apixaban for Reduction in Stroke and Other Thromboembolic Events in Atrial Fibrillation) trial. Eur Heart J. 2016;37(38):2869-2878. doi:10.1093/ eurheartj/ehw124

9. Di Nisio M, Vedovati MC, Riera-Mestre A, et al. Treatment of venous thromboembolism with rivaroxaban in relation to body weight. A sub-analysis of the EINSTEIN DVT/PE studies. Thromb Haemost. 2016;116(4):739-746. doi:10.1160/TH16-02-0087

10. Güler E, Babur güler G, Demir GG, Hatipoğlu S. A review of the fixed dose use of new oral anticoagulants in obese patients: is it really enough? Anatol J Cardiol. 2015;15(12):1020-1029. doi:10.5152/ AnatolJCardiol.2015.6532

11. World Health Organisation. Obesity: preventing and managing the global epidemic. Report of a WHO consultation. World Health Organ Tech Rep Ser. 2000;894:i-253.

12. Guyatt GH, Norris SL, Schulman S, et al. Methodology for the development of antithrombotic therapy and prevention of thrombosis guidelines: antithrombotic therapy and prevention of thrombosis, 9th ed: American College of Chest Physicians evidence-based clinical practice guidelines. Chest. 2012;141(2):53S-70S. doi:10.1378/ chest.11-2288 
13. Spyropoulos AC, Brohi K, Caprini J, et al.; SSC Subcommittee on Perioperative and Critical Care Thrombosis and Haemostasis of the International Society on Thrombosis and Haemostasis. Scientific and Standardization Committee Communication: guidance document on the periprocedural management of patients on chronic oral anticoagulant therapy: recommendations for standardized reporting of procedural/surgical bleed risk and patient-specific thromboembolic risk. J Thromb Haemost. 2019;17 (11):1966-1972.

14. Yao X, Abraham NS, Alexander GC, et al. Effect of adherence to oral anticoagulants on risk of stroke and major bleeding among patients with atrial fibrillation. $J$ Am Heart Assoc. 2016;5(2):e003074. doi:10.1161/JAHA.115.003074

15. Eikelboom JW, Quinlan DJ, Hirsh J, Connolly SJ, Weitz JI. Laboratory monitoring of non-vitamin $\mathrm{k}$ antagonist oral anticoagulant use in patients with atrial fibrillation: a Review. JAMA cardiol. 2017;2(5):566-574. doi:10.1001/jamacardio.2017.0364

16. Uprichard J. Management of rivaroxaban in relation to bodyweight and body mass index. Ther Adv Cardiovasc Dis. 2016;10 (5):294-303. doi:10.1177/1753944716643645

17. Netley J, Howard K, Wilson W. Effects of body mass index on the safety and effectiveness of direct oral anticoagulants: a retrospective review. J Thromb Thrombolysis. 2019;48(3):359-365. doi:10.1007/ s11239-019-01857-2

18. Murakawa $\mathrm{Y}$, Ikeda T, Ogawa $\mathrm{S}$, et al. Impact of body mass index on real-world outcomes of rivaroxaban treatment in Japanese patients with non-valvular atrial fibrillation. Heart Vessels. 2020;35 (8):1125-1134. doi:10.1007/s00380-020-01587-z

19. Tittl L, Endig S, Marten S, Reitter A, Beyer-Westendorf I, BeyerWestendorf J. Impact of BMI on clinical outcomes of DOAC therapy in daily care - results of the prospective Dresden DOAC Registry (NCT01588119). Int $J$ Cardiol. 2018;262:85-91. doi:10.1016/j. ijcard.2018.03.060

20. Echouffo-Tcheugui JB, Xu H, Matsouaka RA, et al. Diabetes and long-term outcomes of ischaemic stroke: findings from get with the guidelines-stroke. Eur Heart J. 2018;39(25):2376-2386. doi:10.1093/ eurheartj/ehy036

21. Schulman S, Kearon C, Kakkar AK, et al. Extended use of dabigatran, warfarin, or placebo in venous thromboembolism. $N$ Engl J Med. 2013;368(8):709-718. doi:10.1056/NEJMoa1113697

22. Kido K, Ngorsuraches S. Comparing the efficacy and safety of direct oral anticoagulants with warfarin in the morbidly obese population with atrial fibrillation. Ann Pharmacother. 2019;53(2):165-170. doi: $10.1177 / 1060028018796604$

23. Piran S, Traquair H, Chan N, Bhagirath V, Schulman S. Peak plasma concentration of direct oral anticoagulants in obese patients weighing over 120 kilograms: a retrospective study. Res Pract Thromb Haemost. 2018;2(4):684-688. doi:10.1002/rth2.12146

24. Di Minno MN, Lupoli R, Di Minno A, Ambrosino P, Scalera A, Dentali F. Effect of body weight on efficacy and safety of direct oral anticoagulants in the treatment of patients with acute venous thromboembolism: a meta-analysis of randomized controlled trials. Ann Med. 2015;47(1):61-68. doi:10.3109/07853890.2014.982064

25. Lee CH, Lin TY, Chang SH, et al. Body mass index is an independent predictor of major bleeding in non-valvular atrial fibrillation patients taking dabigatran. Int J Cardiol. 2017;228:771-778. doi:10.1016/j. ijcard.2016.11.277
26. Koscielny J, Rosenthal C, von Heymann C. Update on direct oral anticoagulants (DOACs). update direkte orale antikoagulanzien (DOAKs) - perioperatives "Switching", Medikamenteninteraktionen und Persistenz. Hamostaseologie. 2017;37(4):267-275. doi:10.5482/ HAMO-16-10-0041

27. Kubitza D, Becka M, Zuehlsdorf M, Mueck W. Body weight has limited influence on the safety, tolerability, pharmacokinetics, or pharmacodynamics of rivaroxaban (BAY 59-7939) in healthy subjects. J Clin Pharmacol. 2007;47(2):218-226. doi:10.1177/ 0091270006296058

28. Kido K, Lee JC, Hellwig T, Gulseth MP. Use of direct oral anticoagulants in morbidly obese patients. Pharmacotherapy. 2020;40 (1):72-83. doi:10.1002/phar.2353

29. Martin AC, Thomas W, Mahir Z, et al. Direct oral anticoagulant concentrations in obese and high body weight patients: a Cohort Study. Thromb Haemost. 2020.

30. Yamashita T, Koretsune Y, Yasaka M, et al. Randomized, multicenter, warfarin-controlled Phase II study of edoxaban in Japanese patients with non-valvular atrial fibrillation. Circ J. 2012;76(8):1840-1847. doi:10.1253/circj.CJ-11-1140

31. Covert K, Branam DL. Direct-acting oral anticoagulant use at extremes of body weight: literature review and recommendations. Am J Health Syst Pharm. 2020;77(11):865-876. doi:10.1093/ajhp/zxaa059

32. Boonyawat K, Caron F, Li A, et al. Association of body weight with efficacy and safety outcomes in Phase III RANDOMIZED controlled trials of direct oral anticoagulants: a systematic review and meta-analysis. J Thromb Haemost. 2017;15(7):1322-1333. doi: $10.1111 /$ jh. 13701

33. Kvasnicka T, Malikova I, Zenahlikova Z, et al. Rivaroxaban Metabolism, pharmacologic properties and drug interactions. Curr Drug Metab. 2017;18(7):636-642. doi:10.2174/ 1389200218666170518165443

34. Hijazi Z, Oldgren J, Lindbäck J, et al. The novel biomarker-based $\mathrm{ABC}$ (age, biomarkers, clinical history)-bleeding risk score for patients with atrial fibrillation: a derivation and validation study. Lancet. 2016;387(10035):2302-2311. doi:10.1016/S0140-6736(16) 00741-8

35. Di Fusco SA, Lucà $F$, Benvenuto $M$, et al. Major bleeding with old and novel oral anticoagulants: how to manage it. Focus on general measures. Int $J$ Cardiol. 2018;387:80-84. doi:10.1016/j. ijcard.2018.05.061

36. Baber U, Mastoris I, Mehran R. Balancing ischaemia and bleeding risks with novel oral anticoagulants. Nat Rev Cardiol. 2014;11 (12):693-703. doi:10.1038/nrcardio.2014.170

37. Kaplan RM, Tanaka Y, Passman RS, et al. Efficacy and safety of direct oral anticoagulants for atrial fibrillation across body mass index categories. $J$ Am Heart Assoc. 2020;9(24):e017383. doi:10.1161/JAHA.120.017383

38. Alma LJ, De Groot C, De Menezes RX, Hermes W, Hordijk PL, Kovačević I. Endothelial dysfunction as a long-term effect of late onset hypertensive pregnancy disorders: high BMI is key. Eur J Obstet Gynecol Reprod Biol. 2018;225:62-69. doi:10.1016/j. ejogrb.2018.04.003

39. Farpour-Lambert NJ, Martin XE, S BDT, et al. Effectiveness of individual and group programmes to treat obesity and reduce cardiovascular disease risk factors in pre-pubertal children. Clin Obes. 2019;9(6):e12335. doi:10.1111/cob.12335 


\section{Publish your work in this journal}

Drug Design, Development and Therapy is an international, peerreviewed open-access journal that spans the spectrum of drug design and development through to clinical applications. Clinical outcomes, patient safety, and programs for the development and effective, safe, and sustained use of medicines are a feature of the journal, which has also been accepted for indexing on PubMed Central. The manuscript management system is completely online and includes a very quick and fair peer-review system, which is all easy to use. Visit http://www. dovepress.com/testimonials.php to read real quotes from published authors.

Submit your manuscript here: https://www.dovepress.com/drug-design-development-and-therapy-journal 